\title{
Notions of generalized $s$-convex functions on fractal sets
}

\author{
Adem Kılıçman ${ }^{1 *}$ and Wedad Saleh ${ }^{2}$
}

${ }^{\text {*Correspondence: }}$ akilic@upm.edu.my

${ }^{1}$ Department of Mathematics and Institute for Mathematical Research, University Putra Malaysia, Serdang, 43400, Malaysia

Full list of author information is available at the end of the article

\begin{abstract}
The purpose of this article is to present some new inequalities for products of generalized convex and generalized s-convex functions on fractal sets. Furthermore, some applications are given.
\end{abstract}

MSC: 26A51;26D07; 26D15; 53C22

Keywords: $s$-convex functions; fractal space; local fractional derivative

\section{Introduction}

Let $f: I \subset \mathbb{R} \longrightarrow \mathbb{R}^{\alpha}$. For any $x_{1}, x_{2} \in I$ and $\gamma \in[0,1]$ if the inequality

$$
f\left(\gamma x_{1}+(1-\gamma) x_{2}\right) \leq \gamma^{\alpha} f\left(x_{1}\right)+(1-\gamma)^{\alpha} f\left(x_{2}\right)
$$

holds, then $f$ is called a generalized convex function on $I$ [1]. In $\alpha=1$, we have convex function, convexity is defined only in geometrical terms as being the property of a function whose graph bears tangents only under it [2].

The convexity of functions plays a significant role in many fields, for example, in biological system, economy, optimization, and so on [3-5].

In recent years, the fractal theory has received significantly remarkable attention from scientists and engineers. In the sense of Mandelbrot, a fractal set is one whose Hausdorff dimension strictly exceeds the topological dimension $[6,7]$. Many researchers studied the properties of functions on fractal space and constructed many kinds of fractional calculus by using different approaches [8-12]. Particularly, in [13], Yang gave the analysis of local fractional functions on fractal space systematically, which includes local fractional calculus and the monotonicity of function.

Let $\mathbb{R}^{\alpha}$ be the real line numbers on fractal space. Then by using Gao-Yang-Kang's concept one can explain the definitions of the local fractional derivative and local fractional integral as in [12-16]. Now if $r_{1}^{\alpha}, r_{2}^{\alpha}$ and $r_{3}^{\alpha} \in \mathbb{R}^{\alpha}(0<\alpha \leq 1)$, then

(1) $r_{1}^{\alpha}+r_{2}^{\alpha} \in \mathbb{R}^{\alpha}, r_{1}^{\alpha} r_{2}^{\alpha} \in \mathbb{R}^{\alpha}$,

(2) $r_{1}^{\alpha}+r_{2}^{\alpha}=r_{2}^{\alpha}+r_{1}^{\alpha}=\left(r_{1}+r_{2}\right)^{\alpha}=\left(r_{2}+r_{1}\right)^{\alpha}$,

(3) $r_{1}^{\alpha}+\left(r_{2}^{\alpha}+r_{3}^{\alpha}\right)=\left(r_{1}^{\alpha}+r_{2}^{\alpha}\right)+r_{3}^{\alpha}$,

(4) $r_{1}^{\alpha} r_{2}^{\alpha}=r_{2}^{\alpha} r_{1}^{\alpha}=\left(r_{1} r_{2}\right)^{\alpha}=\left(r_{2} r_{1}\right)^{\alpha}$,

(5) $r_{1}^{\alpha}\left(r_{2}^{\alpha} r_{3}^{\alpha}\right)=\left(r_{1}^{\alpha} r_{2}^{\alpha}\right) r_{3}^{\alpha}$,

(6) $r_{1}^{\alpha}\left(r_{2}^{\alpha}+r_{3}^{\alpha}\right)=\left(r_{1}^{\alpha} r_{2}^{\alpha}\right)+\left(r_{1}^{\alpha} r_{3}^{\alpha}\right)$,

(c) 2015 Kılıçman and Saleh. This article is distributed under the terms of the Creative Commons Attribution 4.0 International License (http://creativecommons.org/licenses/by/4.0/), which permits unrestricted use, distribution, and reproduction in any medium, provided you give appropriate credit to the original author(s) and the source, provide a link to the Creative Commons license, and indicate if changes were made. 
(7) $r_{1}^{\alpha}+0^{\alpha}=0^{\alpha}+r_{1}^{\alpha}=r_{1}^{\alpha}$ and $r_{1}^{\alpha} \cdot 1^{\alpha}=1^{\alpha} \cdot r_{1}^{\alpha}=r_{1}^{\alpha}$.

Let us start with some definitions as regards the local fractional calculus on $\mathbb{R}^{\alpha}$.

Definition 1.1 [13] Let $y$ be a local fractional continuous function on the interval $\left[a_{1}, a_{2}\right]$. The local fractional integral of the function $y(m)$ of order $\alpha$ is defined by

$$
\begin{aligned}
a_{1} I_{a_{2}}^{(\alpha)} y(m) & =(\Gamma(1+\alpha))^{-1} \int_{a_{1}}^{a_{2}} y(\mu)(d \mu)^{\alpha} \\
& =(\Gamma(1+\alpha))^{-1} \lim _{\Delta \mu \rightarrow 0} \sum_{i=1}^{n} y\left(\mu_{i}\right)\left(\Delta \mu_{i}\right)^{\alpha}
\end{aligned}
$$

with $\Delta \mu_{i}=\mu_{i+1}-\mu_{i}$ and $\Delta \mu=\max \left\{\Delta \mu_{i}: i=1,2, \ldots, n-1\right\}$ where $\left[\mu_{i}, \mu_{i+1}\right], i=0,1, \ldots, n-$ $1, \mu_{0}=a_{1}<\mu_{1}<\cdots<\mu_{n-1}<\mu_{n}=a_{2}$ is a partition of the interval $\left[a_{1}, a_{2}\right]$ and $\Gamma$ is the well-known Gamma function $\Gamma(m)=\int_{0}^{\infty} t^{m-1} e^{-t} d t$.

In [17], Mo and Sui introduced the definitions of two kinds of generalized $s$-convex functions on fractal sets as follows.

\section{Definition 1.2}

(i) A function $f: \mathbb{R}_{+} \longrightarrow \mathbb{R}^{\alpha}$, is called a generalized $s$-convex $(0<s<1)$ in the first sense if

$$
f\left(\gamma_{1} a_{1}+\gamma_{2} a_{2}\right) \leq \gamma_{1}^{s \alpha} f\left(a_{1}\right)+\gamma_{2}^{s \alpha} f\left(a_{2}\right)
$$

for all $a_{1}, a_{2} \in \mathbb{R}_{+}$and all $\gamma_{1}, \gamma_{2} \geq 0$ with $\gamma_{1}^{s}+\gamma_{2}^{s}=1$. This class of functions is denoted by $G K_{s}^{1}$.

(ii) A function $f: \mathbb{R}_{+} \longrightarrow \mathbb{R}^{\alpha}$, is called a generalized $s$-convex $(0<s<1)$ in the second sense if (1) holds for all $a_{1}, a_{2} \in \mathbb{R}_{+}$and all $\gamma_{1}, \gamma_{2} \geq 0$ with $\gamma_{1}+\gamma_{2}=1$. This class of functions is denoted by $G K_{s}^{2}$.

In the same paper, [17], Mo and Sui proved that all functions from $G K_{s}^{2}$, for $s \in(0,1)$, are nonnegative.

In this article, recall that $g s_{1}$ and $g s_{2}$ are the generic classes consistent for those functions that are generalized $s$-convex in the first sense, and in the second sense, respectively. It is well known that there are many important established inequalities for the class of generalized convex functions, however, one of the most famous is known as the generalized Hermit-Hadamard inequality, or the 'generalized Hadamard inequality' and stated as follows (see [1]): let $f$ be a generalized convex function on $\left[a_{1}, a_{2}\right] \subseteq \mathbb{R}, a_{1}<a_{2}$, then

$$
f\left(\frac{a_{1}+a_{2}}{2}\right) \leq \frac{\Gamma(1+\alpha)}{\left(a_{2}-a_{1}\right)^{\alpha}} a_{1} I_{a_{2}}^{(\alpha)} f(x) \leq \frac{f\left(a_{1}\right)+f\left(a_{2}\right)}{2^{\alpha}} .
$$

\section{Basic results for generalized $s$-convex functions}

Lemma 2.1 Iff $\in G K_{s}^{1}$ or $f \in G K_{s}^{2}$, then

$$
f\left(\gamma_{1} x_{1}+\gamma_{2} x_{2}\right) \leq \gamma_{1}^{\alpha s} f\left(x_{1}\right)+\gamma_{2}^{\alpha s} f\left(x_{2}\right)
$$

with $\gamma_{1}, \gamma_{2} \in[0,1]$, exclusively. 
So, we might re-write the definitions of generalized $s$-convexity in the first sense and the second sense as follows.

Definition 2.1 A function $f: I \subset \mathbb{R}_{+} \longrightarrow \mathbb{R}^{\alpha}$ is called a generalized $s$-convex in the first sense if

$$
f\left(\gamma x_{1}+\left(1-\gamma^{s}\right)^{\frac{1}{s}} x_{2}\right) \leq \gamma^{\alpha s} f\left(x_{1}\right)+\left(1-\gamma^{s}\right)^{\alpha} f\left(x_{2}\right)
$$

for all $x_{1}, x_{2} \in I$ and for all $0 \leq \gamma \leq 1$.

Definition 2.2 A function $f: I \subset \mathbb{R}_{+} \longrightarrow \mathbb{R}^{\alpha}$ is called a generalized $s$-convex in the second sense if

$$
f\left(\gamma x_{1}+(1-\gamma) x_{2}\right) \leq \gamma^{\alpha s} f\left(x_{1}\right)+(1-\gamma)^{\alpha s} f\left(x_{2}\right)
$$

for all $x_{1}, x_{2} \in I$ and for all $0 \leq \gamma \leq 1$.

Theorem 2.1 The classes $G K_{1}^{1}, G K_{1}^{2}$ and the class of generalized convex functions are equivalent when the domain is restricted to $\mathbb{R}_{+}$.

Proof Simply an issue of applying the definitions.

Now, some results as regards generalized $s$-convex functions are given.

\section{Remark 2.1}

(i) If $f \in G K_{s}^{1}$, then $f\left(\frac{x_{1}+x_{2}}{2^{\frac{1}{s}}}\right) \leq \frac{f\left(x_{1}\right)+f\left(x_{2}\right)}{2^{\alpha}}$.

(ii) If $f \in G K_{s}^{2}$, then $f\left(\frac{x_{1}+x_{2}}{2}\right) \leq \frac{f\left(x_{1}\right)+f\left(x_{2}\right)}{2^{\alpha s}}$.

(iii) For a function that is both generalized $g s_{1}$ and $g s_{2}$, there is a one-to-one correspondence between the set of all pairs of the sort $(\gamma, \eta)$ with respect to $g s_{1}$ and the set of all pairs of the sort $(\gamma, \eta)$ with respect to $g s_{2}$. (So we may write each $\gamma$ as a $\gamma_{1}^{s}$ and each $\eta$ as a $\eta_{1}^{s}$ and vice versa. This happens in the light of the fact that $0 \leq \gamma, \eta \leq 1,0 \leq s \leq 1$.)

Theorem 2.2 If a function $f \in G K_{s}^{1}$ and $f \in G K_{s}^{2}$, then

$$
f\left(\gamma_{1} x_{1}+\eta_{1} x_{2}\right) \leq \gamma_{1}^{\alpha s} f\left(x_{1}\right)+\eta_{1}^{\alpha s} f\left(x_{2}\right) \leq \gamma_{2}^{\alpha s} f\left(x_{1}\right)+\eta_{2}^{\alpha s} f\left(x_{2}\right)
$$

for some $\left\{\gamma_{i}, \eta_{i}, i=1,2\right\} \subset[0,1]$.

Proof It follows from the one-to-one correspondence proved before. To each $\gamma_{2}, \eta_{2}$ such that $\gamma_{2}+\eta_{2}=1$, there corresponds $\gamma_{1}, \eta_{1}$ where $\gamma_{1}^{s}+\eta_{1}^{s}=1$ and $\gamma_{2} \geq \gamma_{1}, \eta_{2} \geq \eta_{1}$ since $\left\{\gamma_{i}, \eta_{i}, i=1,2\right\} \subset[0,1]$.

Theorem 2.3 If a function $f \in G K_{s}^{1}$ and $f \in G K_{s}^{2}$ and its domain concurs with its counterdomain, then fof $\in G K_{s}^{2}$. 
Proof $f\left(\gamma_{1} x_{1}+\left(1-\gamma_{1}^{s}\right)^{\frac{1}{s}} x_{2}\right) \leq \gamma_{1}^{\alpha s} f\left(x_{1}\right)+\left(1-\gamma_{1}^{s}\right)^{\alpha} f\left(x_{2}\right)$, then

$$
\begin{aligned}
f\left(\gamma_{1}^{\alpha s} f\left(x_{1}\right)+\left(1-\gamma_{1}^{s}\right)^{\alpha} f\left(x_{2}\right)\right) & \leq\left(\gamma_{1}^{\alpha s}\right)^{\alpha s} f\left(f\left(x_{1}\right)\right)+\left(\left(1-\gamma_{1}^{s}\right)^{\alpha}\right)^{\alpha s} f\left(f\left(x_{2}\right)\right) \\
& =\gamma_{2}^{\alpha s} f o f\left(x_{1}\right)+\beta_{2}^{\alpha s} f o f\left(x_{2}\right) .
\end{aligned}
$$

\section{Inequalities for generalized $s$-convex functions}

Theorem 3.1 Let $g:[0,1] \longrightarrow \mathbb{R}^{\alpha}$ be a function such that

$$
g(\gamma)=\frac{1}{\left(a_{2}-a_{1}\right)^{2 \alpha}(\Gamma(1+\alpha))^{2}} \int_{a_{1}}^{a_{2}} \int_{a_{1}}^{a_{2}} f\left(\gamma x_{1}+(1-\gamma) x_{2}\right)\left(d x_{1}\right)^{\alpha}\left(d x_{2}\right)^{\alpha},
$$

where $f:\left[a_{1}, a_{2}\right] \longrightarrow \mathbb{R}^{\alpha}$ and $f \in G K_{s}^{2}$. Then:

(i) $g \in G K_{s}^{2}$ in $[0,1]$. If $f \in G K_{s}^{1}$, then $g \in G K_{s}^{1}$.

(ii) $2^{\alpha} g(0)=2^{\alpha} g(1)=\frac{2^{\alpha}}{\left(a_{2}-a_{1}\right)^{\alpha} \Gamma(1+\alpha)} a_{1} I_{a_{2}}^{(\alpha)} f\left(x_{1}\right)$ is an upper bound for $g(\gamma)$.

(iii) $2^{\alpha(1-s)} g(\gamma) \geq g\left(\frac{1}{2}\right)=\frac{1}{\left(a_{2}-a_{1}\right)^{2 \alpha}(\Gamma(1+\alpha))^{2}} \int_{a_{1}}^{a_{2}} \int_{a_{1}}^{a_{2}} f\left(\frac{x_{1}+x_{2}}{2}\right)\left(d x_{1}\right)^{\alpha}\left(d x_{2}\right)^{\alpha}, \gamma \in[0,1]$.

Proof (i) Take $\left\{\gamma_{1}, \gamma_{2}\right\} \subset[0,1], \gamma_{1}+\gamma_{2}=1, t_{1}, t_{2} \in D$ and $f \in G K_{s}^{2}$, then we have

$$
\begin{aligned}
g\left(\gamma_{1} t_{1}+\gamma_{2} t_{2}\right) & \\
= & \frac{1}{\left(a_{2}-a_{1}\right)^{2 \alpha}(\Gamma(1+\alpha))^{2}} \int_{a_{1}}^{a_{2}} \int_{a_{1}}^{a_{2}} f\left(\left(\gamma_{1} t_{1}+\gamma_{2} t_{2}\right) x_{1}\right. \\
& \left.+\left(1-\left(\gamma_{1} t_{1}+\gamma_{2} t_{2}\right)\right) x_{2}\right)\left(d x_{1}\right)^{\alpha}\left(d x_{2}\right)^{\alpha} \\
\leq & \frac{1}{\left(a_{2}-a_{1}\right)^{2 \alpha}(\Gamma(1+\alpha))^{2}} \int_{a_{1}}^{a_{2}} \int_{a_{1}}^{a_{2}}\left[\gamma_{1}^{\alpha s} f\left(t_{1} x_{1}+x_{2}-t_{1} x_{2}\right)\right. \\
& \left.+\gamma_{2}^{\alpha s} f\left(t_{2} x_{1}+x_{2}-t_{2} x_{2}\right)\right]\left(d x_{1}\right)^{\alpha}\left(d x_{2}\right)^{\alpha} \\
= & \gamma_{1}^{\alpha s} \frac{1}{\left(a_{2}-a_{1}\right)^{2 \alpha}(\Gamma(1+\alpha))^{2}} \int_{a_{1}}^{a_{2}} \int_{a_{1}}^{a_{2}} f\left(t_{1} x_{1}+\left(1-t_{1}\right) x_{2}\right)\left(d x_{1}\right)^{\alpha}\left(d x_{2}\right)^{\alpha} \\
& +\gamma_{2}^{\alpha s} \frac{1}{\left(a_{2}-a_{1}\right)^{2 \alpha}(\Gamma(1+\alpha))^{2}} \int_{a_{1}}^{a_{2}} \int_{a_{1}}^{a_{2}} f\left(t_{2} x_{1}+\left(1-t_{2}\right) x_{2}\right)\left(d x_{1}\right)^{\alpha}\left(d x_{2}\right)^{\alpha} \\
= & \gamma_{1}^{\alpha s} g\left(t_{1}\right)+\gamma_{2}^{\alpha s} g\left(t_{2}\right),
\end{aligned}
$$

which implies that $g \in G K_{s}^{2}$ in $[0,1]$.

(ii) Since $f\left(\gamma x_{1}+(1-\gamma) x_{2}\right) \leq \gamma^{\alpha s} f\left(x_{1}\right)+(1-\gamma)^{\alpha s} f\left(x_{2}\right)$ we have

$$
\begin{aligned}
A= & \frac{1}{(\Gamma(1+\alpha))^{2}} \int_{a_{1}}^{a_{2}} \int_{a_{1}}^{a_{2}} f\left(\gamma x_{1}+(1-\gamma) x_{2}\right)\left(d x_{1}\right)^{\alpha}\left(d x_{2}\right)^{\alpha} \\
\leq & \frac{1}{(\Gamma(1+\alpha))^{2}} \int_{a_{1}}^{a_{2}} \int_{a_{1}}^{a_{2}} \gamma^{\alpha s} f\left(x_{1}\right)\left(d x_{1}\right)^{\alpha}\left(d x_{2}\right)^{\alpha} \\
& +\frac{1}{(\Gamma(1+\alpha))^{2}} \int_{a_{1}}^{a_{2}} \int_{a_{1}}^{a_{2}}(1-\gamma)^{\alpha s} f\left(x_{2}\right)\left(d x_{1}\right)^{\alpha}\left(d x_{2}\right)^{\alpha} \\
= & \gamma^{\alpha s} \frac{1}{\Gamma(1+\alpha)}\left(a_{2}-a_{1}\right)^{\alpha}{ }_{a_{1}} I_{a_{2}}^{(\alpha)} f\left(x_{1}\right)+(1-\gamma)^{\alpha s} \frac{1}{\Gamma(1+\alpha)}\left(a_{2}-a_{1}\right)^{\alpha}{ }_{a_{1}} I_{a_{2}}^{(\alpha)} f\left(x_{2}\right) \\
= & \frac{\left(\gamma^{\alpha s}+(1-\gamma)^{\alpha s}\right)}{\Gamma(1+\alpha)}\left(a_{2}-a_{1}\right)^{\alpha}{ }_{a_{1}} I_{a_{2}}^{(\alpha)} f\left(x_{1}\right),
\end{aligned}
$$


since $\gamma^{\alpha s} \leq 1^{\alpha}$. Because $(1-\gamma)^{\alpha s} \leq 1^{\alpha}$ as well, we have

$$
A \leq \frac{2^{\alpha}}{\Gamma(1+\alpha)}\left(a_{2}-a_{1}\right)^{\alpha}{ }_{a_{1}} I_{a_{2}}^{(\alpha)} f\left(x_{1}\right)
$$

(iii) Since $f$ is generalized $s$-convex in the second sense,

$$
\frac{f\left(\gamma x_{1}+(1-\gamma) x_{2}\right)+f\left((1-\gamma) x_{1}+\gamma x_{2}\right)}{2^{\alpha s}} \geq f\left(\frac{x_{1}+x_{2}}{2}\right)
$$

for all $\gamma \in[0,1]$ and $x_{1}, x_{2} \in[a, b]$.

If we integrate on $\left[a_{1}, a_{2}\right] \times\left[a_{1}, a_{2}\right]$, we obtain

$$
\begin{aligned}
& \frac{1}{2^{\alpha s}(\Gamma(1+\alpha))^{2}} \int_{a_{1}}^{a_{2}} \int_{a_{1}}^{a_{2}}\left[f\left(\gamma x_{1}+(1-\gamma) x_{2}\right)+f\left((1-\gamma) x_{1}+\gamma x_{2}\right)\right]\left(d x_{1}\right)^{\alpha}\left(d x_{2}\right)^{\alpha} \\
& \quad \geq \frac{1}{(\Gamma(1+\alpha))^{2}} \int_{a_{1}}^{a_{2}} \int_{a_{1}}^{a_{2}} f\left(\frac{x_{1}+x_{2}}{2}\right)\left(d x_{1}\right)^{\alpha}\left(d x_{2}\right)^{\alpha} \\
& \quad \Rightarrow \\
& \frac{1}{2^{\alpha(s-1)}\left(a_{2}-a_{1}\right)^{2 \alpha}(\Gamma(1+\alpha))^{2}} \int_{a_{1}}^{a_{2}} \int_{a_{1}}^{a_{2}} f\left(\gamma x_{1}+(1-\gamma) x_{2}\right)\left(d x_{1}\right)^{\alpha}\left(d x_{2}\right)^{\alpha} \\
& \geq \frac{1}{\left(a_{2}-a_{1}\right)^{2 \alpha}(\Gamma(1+\alpha))^{2}} \int_{a_{1}}^{a_{2}} \int_{a_{1}}^{a_{2}} f\left(\frac{x_{1}+x_{2}}{2}\right)\left(d x_{1}\right)^{\alpha}\left(d x_{2}\right)^{\alpha},
\end{aligned}
$$

which means that

$$
2^{\alpha(1-s)} g(\gamma) \geq g\left(\frac{1}{2}\right)
$$

The proof is complete.

Assume the following functions:

(i) $g_{F_{1}}:[0,1] \longrightarrow \mathbb{R}^{\alpha}$, defined by

$$
g_{F_{1}}(\gamma)=\frac{1}{(\Gamma(1+\alpha))^{2}} \int_{a_{1}}^{a_{2}} \int_{a_{1}}^{a_{2}} f\left(\gamma x_{1}+(1-\gamma) x_{2}\right) F\left(x_{1}\right) F\left(x_{2}\right)\left(d x_{1}\right)^{\alpha}\left(d x_{2}\right)^{\alpha},
$$

where $f:\left[a_{1}, a_{2}\right] \subset \mathbb{R}_{+} \longrightarrow \mathbb{R}^{\alpha}$ and $f \in G K_{s}^{1}$.

(ii) $g_{F_{2}}:[0,1] \longrightarrow \mathbb{R}^{\alpha}$, defined by

$$
g_{F_{2}}(\gamma)=\frac{1}{(\Gamma(1+\alpha))^{2}} \int_{a_{1}}^{a_{2}} \int_{a_{1}}^{a_{2}} f\left(\gamma x_{1}+(1-\gamma) x_{2}\right) F\left(x_{1}\right) F\left(x_{2}\right)\left(d x_{1}\right)^{\alpha}\left(d x_{2}\right)^{\alpha},
$$

where $f:\left[a_{1}, a_{2}\right] \subset \mathbb{R}_{+} \longrightarrow \mathbb{R}^{\alpha}$ and $f \in G K_{s}^{2}$.

\section{Theorem 3.2 The following holds:}

(i) $g_{F_{1}}$ and $g_{F_{2}}$ are both symmetric about $\gamma=\frac{1}{2}$.

(ii) $g_{F_{2}} \in G K_{s}^{2}$ in $[0,1]$. 
(iii) We have the upper bound

$$
2^{\alpha} g_{F_{2}}(1)=\frac{2^{\alpha}}{(\Gamma(1+\alpha))^{2}} \int_{a_{1}}^{a_{2}} \int_{a_{1}}^{a_{2}} f\left(x_{1}\right) F\left(x_{1}\right) F\left(x_{2}\right)\left(d x_{1}\right)^{\alpha}\left(d x_{2}\right)^{\alpha}
$$

for the function $g_{F_{2}}(\gamma)$.

Proof (i) $g_{F_{1}}$ and $g_{F_{2}}$ are both symmetric about $\gamma=\frac{1}{2}$ because $g_{F_{1}}(\gamma)=g_{F_{1}}(1-\gamma)$ and $g_{F_{2}}(\gamma)=g_{F_{2}}(1-\gamma)$.

(ii) We get

$$
\begin{aligned}
g_{F_{2}}\left(\gamma t_{1}+(1-\gamma) t_{2}\right)= & \frac{1}{(\Gamma(1+\alpha))^{2}} \int_{a_{1}}^{a_{2}} \int_{a_{1}}^{a_{2}} f\left(\left(\gamma t_{1}+(1-\gamma) t_{2}\right) x_{1}\right. \\
& \left.+\left(1-\left(\gamma t_{1}+(1-\gamma) t_{2}\right) x_{2}\right)\right) F\left(x_{1}\right) F\left(x_{2}\right)\left(d x_{1}\right)^{\alpha}\left(d x_{2}\right)^{\alpha} .
\end{aligned}
$$

But

$$
\begin{aligned}
& \left(\gamma t_{1}+(1-\gamma) t_{2}\right) x_{1}+\left(1-\left(\gamma t_{1}+(1-\gamma) t_{2}\right) x_{2}\right. \\
& \quad=\gamma t_{1} x_{1}+(1-\gamma) t_{2} x_{1}+\gamma x_{2}+(1-\gamma) x_{2}-\gamma t_{1} x_{2}-(1-\gamma) t_{2} x_{2} \\
& \quad=\gamma\left(t_{1} x_{1}+x_{2}-t_{1} x_{2}\right)+(1-\gamma)\left(t_{2} x_{1}+x_{2}-t_{2} x_{2}\right) .
\end{aligned}
$$

Since $f \in G K_{s}^{2}$,

$$
\begin{aligned}
g_{F_{2}}(\gamma & \left.t_{1}+(1-\gamma) t_{2}\right) \\
= & \frac{1}{(\Gamma(1+\alpha))^{2}} \int_{a_{1}}^{a_{2}} \int_{a_{1}}^{a_{2}} f\left(\left(\gamma t_{1}+(1-\gamma) t_{2}\right) x_{1}\right. \\
& \left.+\left(1-\left(\gamma t_{1}+(1-\gamma) t_{2}\right) x_{2}\right)\right) F\left(x_{1}\right) F\left(x_{2}\right)\left(d x_{1}\right) \alpha\left(d x_{2}\right)^{\alpha} \\
\leq & \gamma^{\alpha s} \frac{1}{(\Gamma(1+\alpha))^{2}} \int_{a_{1}}^{a_{2}} \int_{a_{1}}^{a_{2}} f\left(t_{1} x_{1}+\left(1-t_{1}\right) x_{2}\right) F\left(x_{1}\right) F\left(x_{2}\right)\left(d x_{1}\right)^{\alpha}\left(d x_{2}\right)^{\alpha} \\
& +(1-\gamma)^{\alpha s} \frac{1}{(\Gamma(1+\alpha))^{2}} \int_{a_{1}}^{a_{2}} \int_{a_{1}}^{a_{2}} f\left(t_{2} x_{1}+\left(1-t_{2}\right) x_{2}\right) F\left(x_{1}\right) F\left(x_{2}\right)\left(d x_{1}\right)^{\alpha}\left(d x_{2}\right)^{\alpha} \\
= & \gamma^{\alpha s} g_{F_{2}}\left(t_{1}\right)+(1-\gamma)^{\alpha s} g_{F_{2}}\left(t_{2}\right),
\end{aligned}
$$

which proves that $g_{F_{2}} \in G K_{s}^{2}$.

(iii) From the definition and the assumptions, we get

$$
\begin{aligned}
g_{F_{2}}(\gamma)= & \frac{1}{(\Gamma(1+\alpha))^{2}} \int_{a_{1}}^{a_{2}} \int_{a_{1}}^{a_{2}} f\left(\gamma x_{1}+(1-\gamma) x_{2}\right) F\left(x_{1}\right) F\left(x_{2}\right)\left(d x_{1}\right)^{\alpha}\left(d x_{2}\right)^{\alpha} \\
\leq & \gamma^{\alpha s} \frac{1}{(\Gamma(1+\alpha))^{2}} \int_{a_{1}}^{a_{2}} \int_{a_{1}}^{a_{2}} f\left(x_{1}\right) F\left(x_{1}\right) F\left(x_{2}\right)\left(d x_{1}\right)^{\alpha}\left(d x_{2}\right)^{\alpha} \\
& +(1-\gamma)^{\alpha s} \frac{1}{(\Gamma(1+\alpha))^{2}} \int_{a_{1}}^{a_{2}} \int_{a_{1}}^{a_{2}} f\left(x_{2}\right) F\left(x_{1}\right) F\left(x_{2}\right)\left(d x_{1}\right)^{\alpha}\left(d x_{2}\right)^{\alpha} \\
= & \left(\gamma^{\alpha s}+(1-\gamma)^{\alpha s}\right) \frac{1}{(\Gamma(1+\alpha))^{2}} \int_{a_{1}}^{a_{2}} \int_{a_{1}}^{a_{2}} f\left(x_{1}\right) F\left(x_{1}\right) F\left(x_{2}\right)\left(d x_{1}\right)^{\alpha}\left(d x_{2}\right)^{\alpha}
\end{aligned}
$$




$$
\begin{aligned}
& \leq \frac{2^{\alpha}}{(\Gamma(1+\alpha))^{2}} \int_{a_{1}}^{a_{2}} \int_{a_{1}}^{a_{2}} f\left(x_{1}\right) F\left(x_{1}\right) F\left(x_{2}\right)\left(d x_{1}\right)^{\alpha}\left(d x_{2}\right)^{\alpha} \\
& =2^{\alpha} g_{F_{2}}(1) .
\end{aligned}
$$

Theorem 3.3 Let $f_{1}, f_{2}:\left[a_{1}, a_{2}\right] \subset \mathbb{R}_{+} \longrightarrow \mathbb{R}^{\alpha}, a_{1}<a_{2}$, be nonnegative, and generalized sconvex functions in the second sense. If $f_{1} \in G K_{s_{1}}^{2}$ and $f_{2} \in G K_{s_{2}}^{2}$ on $\left[a_{1}, a_{2}\right]$ for some $\gamma \in[0,1]$ and $s_{1}, s_{2} \in(0,1]$, then

$$
\begin{aligned}
& \frac{1}{(\Gamma(1+\alpha))^{3}} \int_{a_{1}}^{a_{2}} \int_{a_{1}}^{a_{2}} \int_{0}^{1} f_{1}\left(\gamma x_{1}+(1-\gamma) x_{2}\right) f_{2}\left(\gamma x_{1}+(1-\gamma) x_{2}\right)(d \gamma)^{\alpha}\left(d x_{2}\right)^{\alpha}\left(d x_{1}\right)^{\alpha} \\
& \leq \frac{2^{\alpha} \Gamma\left(1+\left(\gamma_{1}+\gamma_{2}\right) \alpha\right)}{\Gamma\left(1+\left(\gamma_{1}+\gamma_{2}+1\right) \alpha\right)} \frac{1}{(\Gamma(1+\alpha))^{2}}\left(a_{2}-a_{1}\right)^{\alpha} \int_{a_{1}}^{a_{2}} f_{1}\left(x_{1}\right) f_{2}\left(x_{1}\right)\left(d x_{1}\right)^{\alpha} \\
& \quad+\frac{1}{2^{\alpha}(\Gamma(1+\alpha))^{3}} \beta_{\alpha}\left(a_{2}-a_{1}\right)^{2 \alpha}\left[T_{1}(a, b)+T_{2}(a, b)\right],
\end{aligned}
$$

where

$$
\begin{aligned}
& \beta_{\alpha}=\int_{0}^{1} \gamma^{\alpha s_{1}}(1-\gamma)^{\alpha s_{2}}(d \gamma)^{\alpha}, \\
& T_{1}\left(a_{1}, a_{2}\right)=f_{1}\left(a_{1}\right) f_{2}\left(a_{1}\right)+f_{1}\left(a_{2}\right) f_{2}\left(a_{2}\right),
\end{aligned}
$$

and

$$
T_{2}\left(a_{1}, a_{2}\right)=f_{1}\left(a_{1}\right) f_{2}\left(a_{2}\right)+f_{1}\left(a_{2}\right) f_{2}\left(a_{1}\right) .
$$

Proof Since $f_{1} \in G K_{s_{1}}^{2}$ and $f_{2} \in G K_{s_{2}}^{2}$ on $\left[a_{1}, a_{2}\right]$,

$$
\begin{aligned}
& f_{1}\left(\gamma x_{1}+(1-\gamma) x_{2}\right) \leq \gamma^{\alpha s_{1}} f_{1}\left(x_{1}\right)+(1-\gamma)^{\alpha s_{1}} f_{1}\left(x_{2}\right), \\
& f_{2}\left(\gamma x_{1}+(1-\gamma) x_{2}\right) \leq \gamma^{\alpha s_{2}} f_{2}\left(x_{1}\right)+(1-\gamma)^{\alpha s_{2}} f_{2}\left(x_{2}\right),
\end{aligned}
$$

for all $\gamma \in[0,1]$. Since $f_{1}$ and $f_{1}$ are nonnegative,

$$
\begin{aligned}
& f_{1}\left(\gamma x_{1}+(1-\gamma) x_{2}\right) f_{2}\left(\gamma x_{1}+(1-\gamma) x_{2}\right) \\
& \quad \leq \gamma^{\alpha\left(s_{1}+s_{2}\right)} f_{1}\left(x_{1}\right) f_{2}\left(x_{1}\right)+(1-\gamma)^{\alpha\left(s_{1}+s_{2}\right)} f_{1}\left(x_{2}\right) f_{2}\left(x_{2}\right) \\
& \quad+\gamma^{\alpha s_{1}}(1-\gamma)^{\alpha s_{2}} f_{1}\left(x_{1}\right) f_{2}\left(x_{2}\right)+\gamma^{\alpha s_{2}}(1-\gamma)^{\alpha s_{1}} f_{1}\left(x_{2}\right) f_{2}\left(x_{1}\right) .
\end{aligned}
$$

Integrating both sides of the above inequality over $[0,1]$, we obtain

$$
\begin{aligned}
& \frac{1}{\Gamma(1+\alpha)} \int_{0}^{1} f_{1}\left(\gamma x_{1}+(1-\gamma) x_{2}\right) f_{2}\left(\gamma x_{1}+(1-\gamma) x_{2}\right)(d \gamma)^{\alpha} \\
& \leq \frac{1}{\Gamma(1+\alpha)} f_{1}\left(x_{1}\right) f_{2}\left(x_{1}\right) \int_{0}^{1} \gamma^{\alpha\left(s_{1}+s_{2}\right)}(d \gamma)^{\alpha} \\
& \quad+\frac{1}{\Gamma(1+\alpha)} f_{1}\left(x_{2}\right) f_{2}\left(x_{2}\right) \int_{0}^{1}(1-\gamma)^{\alpha\left(s_{1}+s_{2}\right)}(d \gamma)^{\alpha} \\
& \quad+\frac{1}{\Gamma(1-\alpha)} f_{1}\left(x_{1}\right) f_{2}\left(x_{2}\right) \int_{0}^{1} \gamma^{\alpha s_{1}}(1-\gamma)^{\alpha s_{2}}(d \gamma)^{\alpha}
\end{aligned}
$$




$$
\begin{aligned}
& +\frac{1}{\Gamma(1+\alpha)} f_{1}\left(x_{2}\right) f_{2}\left(x_{1}\right) \int_{0}^{1} \gamma^{\alpha s_{2}}(1-\gamma)^{\alpha s_{1}}(d \gamma)^{\alpha} \\
= & \frac{\Gamma\left(1+\left(s_{1}+s_{2}\right) \alpha\right)}{\Gamma\left(1+\left(s_{1}+s_{2}+1\right) \alpha\right)} f_{1}\left(x_{1}\right) f_{2}\left(x_{1}\right)+\frac{\Gamma\left(1+\left(s_{1}+s_{2}\right) \alpha\right)}{\Gamma\left(1+\left(s_{1}+s_{2}+1\right) \alpha\right)} f_{1}\left(x_{2}\right) f_{2}\left(x_{2}\right) \\
& +\frac{1}{\Gamma(1+\alpha)} \beta_{\alpha}\left[f_{1}\left(x_{1}\right) f_{2}\left(x_{2}\right)+f_{1}\left(x_{2}\right) f_{2}\left(x_{1}\right)\right] .
\end{aligned}
$$

If we integrate both sides of the above inequalities on $\left[a_{1}, a_{2}\right] \times\left[a_{1}, a_{2}\right]$, we obtain

$$
\begin{aligned}
& \frac{1}{(\Gamma(1+\alpha))^{3}} \int_{a_{1}}^{a_{2}} \int_{a_{1}}^{a_{2}} \int_{0}^{1} f_{1}\left(\gamma x_{1}+(1-\gamma) x_{2}\right) f_{2}\left(\gamma x_{1}+(1-\gamma) x_{2}\right)(d \gamma)^{\alpha}\left(d x_{2}\right)^{\alpha}\left(d x_{1}\right)^{\alpha} \\
& \leq \frac{\Gamma\left(1+\left(s_{1}+s_{2}\right) \alpha\right)}{\Gamma\left(1+\left(s_{1}+s_{2}+1\right) \alpha\right)} \frac{1}{(\Gamma(1+\alpha))^{2}}\left[\int_{a_{1}}^{a_{2}} \int_{a_{1}}^{a_{2}} f_{1}\left(x_{1}\right) f_{2}\left(x_{1}\right)\left(d x_{2}\right)^{\alpha}\left(d x_{1}\right)^{\alpha}\right. \\
& \left.+\int_{a_{1}}^{a_{2}} \int_{a_{1}}^{a_{2}} f_{1}\left(x_{2}\right) f_{2}\left(x_{2}\right)\left(d x_{2}\right)^{\alpha}\left(d x_{1}\right)^{\alpha}\right] \\
& +\frac{1}{(\Gamma(1+\alpha))^{3}} \beta_{\alpha}\left[\int_{a_{1}}^{a_{2}} \int_{a_{1}}^{a_{2}} f_{1}\left(x_{1}\right) f_{2}\left(x_{2}\right)\left(d x_{2}\right)^{\alpha}\left(d x_{1}\right)^{\alpha}\right. \\
& \left.+\int_{a_{1}}^{a_{2}} \int_{a_{1}}^{a_{2}} f_{1}\left(x_{2}\right) f_{2}\left(x_{1}\right)\left(d x_{2}\right)^{\alpha}\left(d x_{1}\right)^{\alpha}\right] \\
& =\frac{\Gamma\left(1+\left(s_{1}+s_{2}\right) \alpha\right)}{\Gamma\left(1+\left(s_{1}+s_{2}+1\right) \alpha\right)} \frac{1}{(\Gamma(1+\alpha))^{2}}\left(a_{2}-a_{1}\right)^{\alpha} \\
& \times\left[\int_{a_{1}}^{a_{2}} f_{1}\left(x_{1}\right) f_{2}\left(x_{1}\right)\left(d x_{1}\right)^{\alpha}+\int_{a_{1}}^{a_{2}} f_{1}\left(x_{2}\right) f_{2}\left(x_{2}\right)\left(d x_{2}\right)^{\alpha}\right] \\
& +\frac{1}{(\Gamma(1+\alpha))^{3}} \beta_{\alpha}\left[\int_{a_{1}}^{a_{2}} f_{1}\left(x_{1}\right)\left(d x_{1}\right)^{\alpha} \int_{a_{1}}^{a_{2}} f_{2}\left(x_{2}\right)\left(d x_{2}\right)^{\alpha}\right. \\
& \left.+\int_{a_{1}}^{a_{2}} f_{1}\left(x_{2}\right)\left(d x_{2}\right)^{\alpha} \int_{a_{1}}^{a_{2}} f_{2}\left(x_{1}\right)\left(d x_{1}\right)^{\alpha}\right] \text {. }
\end{aligned}
$$

By using the right half of the generalized Hadamard inequality on the right side of the above inequality, we have

$$
\begin{aligned}
& \frac{1}{(\Gamma(1+\alpha))^{3}} \int_{a_{1}}^{a_{2}} \int_{a_{1}}^{a_{2}} \int_{0}^{1} f_{1}\left(\gamma x_{1}+(1-\gamma) x_{2}\right) f_{2}\left(\gamma x_{1}+(1-\gamma) x_{2}\right)(d \gamma)^{\alpha}\left(d x_{2}\right)^{\alpha}\left(d x_{1}\right)^{\alpha} \\
& \leq \frac{2^{\alpha} \Gamma\left(1+\left(s_{1}+s_{2}\right) \alpha\right)}{\Gamma\left(1+\left(s_{1}+s_{2}+1\right) \alpha\right)} \frac{1}{(\Gamma(1+\alpha))^{2}}\left(a_{2}-a_{1}\right)^{\alpha} \int_{a_{1}}^{a_{2}} f_{1}\left(x_{1}\right) f_{2}\left(x_{1}\right)\left(d x_{1}\right)^{\alpha} \\
& \quad+\frac{1}{2^{\alpha}(\Gamma(1+\alpha))^{3}} \beta_{\alpha}\left(a_{2}-a_{1}\right)^{2 \alpha}\left[T_{1}\left(a_{1}, a_{2}\right)+T_{2}\left(a_{1}, a_{2}\right)\right] .
\end{aligned}
$$

The proof is complete.

\section{Remark 3.1}

(i) If $\alpha=1$, in Theorem 3.3, then

$$
\beta=\int_{0}^{1} \gamma^{s_{1}}(1-\gamma)^{s_{2}}(d \gamma)=\frac{\Gamma\left(s_{1}+1\right) \Gamma\left(s_{2}+1\right)}{\Gamma\left(s_{1}+s_{2}+2\right)}
$$


and

$$
\begin{aligned}
\int_{a_{1}}^{a_{2}} \int_{a_{1}}^{a_{2}} \int_{0}^{1} f_{1}\left(\gamma x_{1}+(1-\gamma) x_{2}\right) f_{2}\left(\gamma x_{1}+(1-\gamma) x_{2}\right)(d \gamma)\left(d x_{1}\right)\left(d x_{2}\right) \\
\leq \frac{2}{s_{1}+s_{2}+1}\left(a_{2}-a_{1}\right) \int_{a_{1}}^{a_{2}} f_{1}\left(x_{1}\right) f_{2}\left(x_{1}\right) d x_{1} \\
\quad+\frac{\Gamma\left(s_{1}+1\right) \Gamma\left(s_{2}+1\right)}{2 \Gamma\left(s_{1}+s_{2}+2\right)}\left(a_{2}-a_{1}\right)^{2}\left[T_{1}\left(a_{1}, a_{2}\right)+T_{2}\left(a_{1}, a_{2}\right)\right] .
\end{aligned}
$$

(ii) If $\alpha=1$, and $s_{1}=s_{2}=1$, then $\frac{\Gamma\left(s_{1}+1\right) \Gamma\left(s_{2}+1\right)}{\Gamma\left(s_{1}+s_{2}+2\right)}=\frac{1}{6}$. Also,

$$
\beta=\int_{0}^{1} \gamma^{s_{1}}(1-\gamma)^{s_{2}}(d \gamma)=\frac{\Gamma\left(s_{1}+1\right) \Gamma\left(s_{2}+1\right)}{\Gamma\left(s_{1}+s_{2}+2\right)},
$$

which implies that

$$
\begin{aligned}
\int_{a_{1}}^{a_{2}} \int_{a_{1}}^{a_{2}} \int_{0}^{1} f_{1}\left(\gamma x_{1}+(1-\gamma) x_{2}\right) f_{2}\left(\gamma x_{1}+(1-\gamma) x_{2}\right)(d \gamma)\left(d x_{2}\right)\left(d x_{1}\right) \\
\leq \frac{2}{3}\left(a_{2}-a_{1}\right) \int_{a_{1}}^{a_{2}} f_{1}\left(x_{1}\right) f_{2}\left(x_{1}\right) d x_{1} \\
\quad+\frac{1}{12}\left(a_{2}-a_{1}\right)^{2}\left[T_{1}\left(a_{1}, a_{2}\right)+T_{2}\left(a_{1}, a_{2}\right)\right] .
\end{aligned}
$$

Theorem 3.4 Let $f_{1}, f_{2}:\left[a_{1}, a_{2}\right] \subset \mathbb{R}_{+} \longrightarrow \mathbb{R}^{\alpha}, a_{1}<a_{2}$, be nonnegative and generalized sconvex functions in the second sense. If $f_{1} \in G K_{s_{1}}^{2}$ and $f_{2} \in G K_{s_{2}}^{2}$ on $\left[a_{1}, a_{2}\right]$ for some $\gamma \in[0,1]$ and $s_{1}, s_{2} \in(0,1]$, then

$$
\begin{aligned}
& \frac{1}{(\Gamma(1+\alpha))^{2}} \int_{a_{1}}^{a_{2}} \int_{0}^{1} f_{1}\left(\gamma x_{1}+(1-\gamma) \frac{a+b}{2}\right) f_{2}\left(\gamma x_{1}+(1-\gamma) \frac{a+b}{2}\right)(d \gamma)^{\alpha}\left(d x_{1}\right)^{\alpha} \\
& \leq \frac{\Gamma\left(1+\left(s_{1}+s_{2}\right) \alpha\right)}{\Gamma\left(1+\left(s_{1}+s_{2}+1\right) \alpha\right)} \frac{1}{\Gamma(1+\alpha)} \int_{a_{1}}^{a_{2}} f_{1}\left(x_{1}\right) f_{2}\left(x_{1}\right)\left(d x_{1}\right)^{\alpha} \\
& \quad+\frac{1}{2^{\alpha} \Gamma(1+\alpha)}\left[\frac{\Gamma\left(1+\left(s_{1}+s_{2}\right) \alpha\right)}{2^{\alpha} \Gamma\left(1+\left(s_{1}+s_{2}+1\right) \alpha\right)}+\frac{1}{\Gamma(1+\alpha)} \beta_{\alpha}\right] \\
& \quad \times\left(a_{2}-a_{1}\right)^{\alpha}\left[T_{1}\left(a_{1}, a_{2}\right)+T_{2}\left(a_{1}, a_{2}\right)\right]
\end{aligned}
$$

where $\beta_{\alpha}, T_{1}\left(a_{1}, a_{2}\right)$, and $T_{2}\left(a_{1}, a_{2}\right)$ are defined in Theorem 3.3 .

Proof Since $f_{1} \in G K_{s_{1}}^{2}$ and $f_{2} \in G K_{s_{2}}^{2}$ on $\left[a_{1}, a_{2}\right]$,

$$
\begin{aligned}
& f_{1}\left(\gamma x_{1}+(1-\gamma) \frac{a_{1}+a_{2}}{2}\right) \leq \gamma^{\alpha s_{1}} f_{1}\left(x_{1}\right)+(1-\gamma)^{\alpha s_{1}} f_{1}\left(\frac{a_{1}+a_{2}}{2}\right) \\
& f_{2}\left(\gamma x_{1}+(1-\gamma) \frac{a_{1}+a_{2}}{2}\right) \leq \gamma^{\alpha s_{2}} f_{2}\left(x_{1}\right)+(1-\gamma)^{\alpha s_{2}} f_{2}\left(\frac{a_{1}+a_{2}}{2}\right)
\end{aligned}
$$

for all $x_{1} \in\left[a_{1}, a_{2}\right]$ and all $\gamma \in[0,1]$. Because $f_{1}$ and $f_{1}$ are nonnegative, we have 


$$
\begin{aligned}
& f_{1}\left(\gamma x_{1}+(1-\gamma) \frac{a_{1}+a_{2}}{2}\right) f_{2}\left(\gamma x_{1}+(1-\gamma) \frac{a_{1}+a_{2}}{2}\right) \\
& \quad \leq \gamma^{\alpha\left(s_{1}+s_{2}\right)} f_{1}\left(x_{1}\right) f_{2}\left(x_{1}\right)+(1-\gamma)^{\alpha\left(s_{1}+s_{2}\right)} f_{1}\left(\frac{a_{1}+a_{2}}{2}\right) f_{2}\left(\frac{a_{1}+a_{2}}{2}\right) \\
& \quad+\gamma^{\alpha s_{1}}(1-\gamma)^{\alpha s_{2}} f_{1}\left(x_{1}\right) f_{2}\left(\frac{a_{1}+a_{2}}{2}\right)+\gamma^{\alpha s_{2}}(1-\gamma)^{\alpha s_{1}} f_{1}\left(\frac{a_{1}+a_{2}}{2}\right) f_{2}\left(x_{1}\right) .
\end{aligned}
$$

Integrating both sides of the above inequality over $[0,1]$, we obtain

$$
\begin{aligned}
& \frac{1}{\Gamma(1+\alpha)} \int_{0}^{1} f_{1}\left(\gamma x_{1}+(1-\gamma) \frac{a_{1}+a_{2}}{2}\right) f_{2}\left(\gamma x_{1}+(1-\gamma) \frac{a_{1}+a_{2}}{2}\right)(d \gamma)^{\alpha} \\
& \leq \frac{1}{\Gamma(1+\alpha)} f_{1}\left(x_{1}\right) f_{2}\left(x_{1}\right) \int_{0}^{1} \gamma^{\alpha\left(s_{1}+s_{2}\right)}(d \gamma)^{\alpha} \\
&+\frac{1}{\Gamma(1+\alpha)} f_{1}\left(\frac{a_{1}+a_{2}}{2}\right) f_{2}\left(\frac{a_{1}+a_{2}}{2}\right) \int_{0}^{1}(1-\gamma)^{\alpha\left(s_{1}+s_{2}\right)}(d \gamma)^{\alpha} \\
&+\frac{1}{\Gamma(1+\alpha)} f_{1}\left(x_{1}\right) f_{2}\left(\frac{a_{1}+a_{2}}{2}\right) \int_{0}^{1} \gamma^{\alpha s_{1}}(1-\gamma)^{\alpha s_{2}}(d \gamma)^{\alpha} \\
&+\frac{1}{\Gamma(1+\alpha)} f_{1}\left(\frac{a_{1}+a_{2}}{2}\right) f_{2}\left(x_{1}\right) \int_{0}^{1} \gamma^{\alpha s_{2}}(1-\gamma)^{\alpha s_{1}}(d \gamma)^{\alpha} \\
&= \frac{\Gamma\left(1+\left(s_{1}+s_{2}\right) \alpha\right)}{\Gamma\left(1+\left(s_{1}+s_{2}+1\right) \alpha\right)} f_{1}\left(x_{1}\right) f_{2}\left(x_{1}\right) \\
&+\frac{\Gamma\left(1+\left(s_{1}+s_{2}\right) \alpha\right)}{\Gamma\left(1+\left(s_{1}+s_{2}+1\right) \alpha\right)} f_{1}\left(\frac{a_{1}+a_{2}}{2}\right) f_{2}\left(\frac{a_{1}+a_{2}}{2}\right) \\
&+\frac{1}{\Gamma(1+\alpha)} \beta_{\alpha}\left[f_{1}\left(x_{1}\right) f_{2}\left(\frac{a_{1}+a_{2}}{2}\right)+f_{1}\left(\frac{a_{1}+a_{2}}{2}\right) f_{2}\left(x_{1}\right)\right]
\end{aligned}
$$

If we integrate both sides of the above inequalities on $\left[a_{1}, a_{2}\right]$, we obtain

$$
\begin{aligned}
& \frac{1}{(\Gamma(1+\alpha))^{2}} \int_{a_{1}}^{a_{2}} \int_{0}^{1} f_{1}\left(\gamma x_{1}+(1-\gamma) \frac{a_{1}+a_{2}}{2}\right) f_{2}\left(\gamma x_{1}+(1-\gamma) \frac{a_{1}+a_{2}}{2}\right)(d \gamma)^{\alpha}\left(d x_{1}\right)^{\alpha} \\
& \leq \frac{\Gamma\left(1+\left(s_{1}+s_{2}\right) \alpha\right)}{\Gamma\left(1+\left(s_{1}+s_{2}+1\right) \alpha\right)} \frac{1}{\Gamma(1+\alpha)} \int_{a_{1}}^{a_{2}} f_{1}\left(x_{1}\right) f_{2}\left(x_{1}\right)\left(d x_{1}\right)^{\alpha} \\
&+\frac{\Gamma\left(1+\left(s_{1}+s_{2}\right) \alpha\right)}{\Gamma\left(1+\left(s_{1}+s_{2}+1\right) \alpha\right)} \frac{1}{\Gamma(1+\alpha)}\left(a_{2}-a_{1}\right)^{\alpha} f_{1}\left(\frac{a_{1}+a_{2}}{2}\right) f_{2}\left(\frac{a_{1}+a_{2}}{2}\right) \\
&+\frac{1}{(\Gamma(1+\alpha))^{2}} \beta_{\alpha}\left[f_{2}\left(\frac{a_{1}+a_{2}}{2}\right) \int_{a_{1}}^{a_{2}} f_{1}\left(x_{1}\right)\left(d x_{1}\right)^{\alpha}+f_{1}\left(\frac{a_{1}+a_{2}}{2}\right) \int_{a_{1}}^{a_{2}} f_{2}\left(x_{1}\right)\left(d x_{1}\right)^{\alpha}\right] \\
& \leq \frac{\Gamma\left(1+\left(s_{1}+s_{2}\right) \alpha\right)}{\Gamma\left(1+\left(s_{1}+s_{2}+1\right) \alpha\right)} \frac{1}{\Gamma(1+\alpha)} \int_{a_{1}}^{a_{2}} f_{1}\left(x_{1}\right) f_{2}\left(x_{1}\right)\left(d x_{1}\right)^{\alpha} \\
&+\frac{\Gamma\left(1+\left(s_{1}+s_{2}\right) \alpha\right)}{\Gamma\left(1+\left(s_{1}+s_{2}+1\right) \alpha\right)} \frac{1}{\Gamma(1+\alpha)}\left(a_{2}-a_{1}\right)^{\alpha} \frac{f_{1}\left(a_{1}\right)+f_{1}\left(a_{2}\right)}{2^{\alpha}} \frac{f_{2}\left(a_{1}\right)+f_{2}\left(a_{2}\right)}{2^{\alpha}} \\
&+\frac{1}{(\Gamma(1+\alpha))^{2}}\left(a_{2}-a_{1}\right)^{\alpha} \beta_{\alpha}\left[2^{\alpha} \frac{f_{1}\left(a_{1}\right)+f_{1}\left(a_{2}\right)}{2^{\alpha}} \frac{f_{2}\left(a_{1}\right)+f_{2}\left(a_{2}\right)}{2^{\alpha}}\right] \\
&= \frac{\Gamma\left(1+\left(s_{1}+s_{2}\right) \alpha\right)}{\Gamma\left(1+\left(s_{1}+s_{2}+1\right) \alpha\right)} \frac{1}{\Gamma(1+\alpha)} \int_{a_{1}}^{a_{2}} f_{1}\left(x_{1}\right) f_{2}\left(x_{1}\right)\left(d x_{1}\right)^{\alpha}
\end{aligned}
$$




$$
\begin{aligned}
& +\frac{\Gamma\left(1+\left(s_{1}+s_{2}\right) \alpha\right)}{4^{\alpha} \Gamma\left(1+\left(s_{1}+s_{2}+1\right) \alpha\right)} \frac{1}{\Gamma(1+\alpha)}\left(a_{2}-a_{1}\right)^{\alpha}\left[T_{1}\left(a_{1}, a_{2}\right)+T_{2}\left(a_{1}, a_{2}\right)\right] \\
& +\frac{1}{2^{\alpha}(\Gamma(1+\alpha))^{2}}\left(a_{2}-a_{1}\right)^{\alpha} \beta_{\alpha}\left[T_{1}\left(a_{1}, a_{2}\right)+T_{2}\left(a_{1}, a_{2}\right)\right] .
\end{aligned}
$$

Then

$$
\begin{aligned}
& \frac{1}{(\Gamma(1+\alpha))^{2}} \int_{a_{1}}^{a_{2}} \int_{0}^{1} f_{1}\left(\gamma x_{1}+(1-\gamma) \frac{a_{1}+a_{2}}{2}\right) f_{2}\left(\gamma x_{1}+(1-\gamma) \frac{a_{1}+a_{2}}{2}\right)(d \gamma)^{\alpha}\left(d x_{1}\right)^{\alpha} \\
& \leq \frac{\Gamma\left(1+\left(s_{1}+s_{2}\right) \alpha\right)}{\Gamma\left(1+\left(s_{1}+s_{2}+1\right)\right) \alpha} \frac{1}{\Gamma(1+\alpha)} \int_{a_{1}}^{a_{2}} f_{1}\left(x_{1}\right) f_{2}\left(x_{1}\right)\left(d x_{1}\right)^{\alpha} \\
& \quad+\frac{1}{2^{\alpha} \Gamma(1+\alpha)}\left[\frac{\Gamma\left(1+\left(s_{1}+s_{2}\right) \alpha\right)}{2^{\alpha} \Gamma\left(1+\left(s_{1}+s_{2}+1\right)\right) \alpha}+\frac{1}{\Gamma(1+\alpha)} \beta_{\alpha}\right] \\
& \quad \times\left(a_{2}-a_{1}\right)^{\alpha}\left[T_{1}\left(a_{1}, a_{2}\right)+T_{2}\left(a_{1}, a_{2}\right)\right] .
\end{aligned}
$$

The proof is complete.

Remark 3.2 If $\alpha=1$, then

$$
\begin{aligned}
\int_{a_{1}}^{a_{2}} & \int_{0}^{1} f_{1}\left(\gamma x_{1}+(1-\gamma) \frac{a_{1}+a_{2}}{2}\right) f_{2}\left(\gamma x_{1}+(1-\gamma) \frac{a_{1}+a_{2}}{2}\right)(d \gamma)\left(d x_{1}\right) \\
\leq & \frac{1}{s_{1}+s_{2}+1} \int_{a_{1}}^{a_{2}} f_{1}\left(x_{1}\right) f_{2}\left(x_{1}\right)\left(d x_{1}\right) \\
& +\frac{1}{2}\left[\frac{1}{2\left(s_{1}+s_{2}+1\right)}+\frac{\Gamma\left(s_{1}+1\right) \Gamma\left(s_{2}+1\right)}{\Gamma\left(s_{1}+s_{2}+1\right)}\right]\left(a_{2}-a_{1}\right)\left[T_{1}\left(a_{1}, a_{2}\right)+T_{2}\left(a_{1}, a_{2}\right)\right] .
\end{aligned}
$$

Theorem 3.5 Let $f_{1}, f_{2}:\left[a_{1}, a_{2}\right] \subset \mathbb{R}_{+} \longrightarrow \mathbb{R}^{\alpha}, a_{1}<a_{2}$, be nonnegative and generalized sconvex functions in the second sense. Iff $\in G K_{s_{1}}^{2}$ and $f_{2} \in G K_{s_{2}}^{2}$ on $\left[a_{1}, a_{2}\right]$ for some $\gamma \in[0,1]$ and $s_{1}, s_{2} \in(0,1]$, then

$$
\begin{aligned}
& \frac{1}{(\Gamma(1+\alpha))^{2}} \int_{a_{1}}^{a_{2}} \int_{0}^{1} f_{1}\left(\gamma \frac{a_{1}+a_{2}}{2}+(1-\gamma) x_{2}\right) f_{2}\left(\gamma \frac{a_{1}+a_{2}}{2}+(1-\gamma) x_{2}\right)(d \gamma)^{\alpha}\left(d x_{2}\right)^{\alpha} \\
& \leq \frac{\Gamma\left(1+\left(s_{1}+s_{2}\right) \alpha\right)}{\Gamma\left(1+\left(s_{1}+s_{2}+1\right) \alpha\right)} \frac{1}{\Gamma(1+\alpha)} \int_{a_{1}}^{a_{2}} f_{1}\left(x_{2}\right) f_{2}\left(x_{2}\right)\left(d x_{2}\right)^{\alpha} \\
& \quad+\frac{1}{2^{\alpha} \Gamma(1+\alpha)}\left[\frac{\Gamma\left(1+\left(s_{1}+s_{2}\right) \alpha\right)}{2^{\alpha} \Gamma\left(1+\left(s_{1}+s_{2}+1\right) \alpha\right)}+\frac{1}{\Gamma(1+\alpha)} \beta_{\alpha}\right] \\
& \quad \times\left(a_{2}-a_{1}\right)^{\alpha}\left[T_{1}\left(a_{1}, a_{2}\right)+T_{2}\left(a_{1}, a_{2}\right)\right],
\end{aligned}
$$

where $\beta_{\alpha}, T_{1}\left(a_{1}, a_{2}\right)$ and $T_{2}\left(a_{1}, a_{2}\right)$ are defined in Theorem 3.3.

Proof The proof of this theorem can easily be given like in Theorem 3.3.

Theorem 3.6 Let $f_{1}, f_{2}:\left[a_{1}, a_{2}\right] \subset \mathbb{R}_{+} \rightarrow \mathbb{R}^{\alpha}, a_{1}<a_{2}$, be nonnegative, and generalized convex functions. If $f_{1}$ and $f_{2}$ are generalized convex on $\left[a_{1}, a_{2}\right]$ for some $\gamma \in[0,1]$, then 


$$
\begin{aligned}
& \frac{1}{(\Gamma(1+\alpha))^{2}} \int_{a_{1}}^{a_{2}} \int_{0}^{1} f_{1}\left(\gamma \frac{a_{1}+a_{2}}{2}+(1-\gamma) x_{2}\right) f_{2}\left(\gamma \frac{a_{1}+a_{2}}{2}+(1-\gamma) x_{2}\right)(d \gamma)^{\alpha}\left(d x_{2}\right)^{\alpha} \\
& \leq \frac{\Gamma(1+2 \alpha)}{\Gamma(1+3 \alpha)} \frac{1}{\Gamma(1+\alpha)} \int_{a_{1}}^{a_{2}} f_{1}\left(x_{2}\right) f_{2}\left(x_{2}\right)\left(d x_{2}\right)^{\alpha} \\
& \quad+\frac{1}{2^{\alpha} \Gamma(1+\alpha)}\left[\frac{\Gamma(1+2 \alpha)}{2^{\alpha} \Gamma(1+3 \alpha)}+\frac{1}{\Gamma(1+\alpha)} \beta_{\alpha}\right]\left(a_{2}-a_{1}\right)^{\alpha}\left[T_{1}\left(a_{1}, a_{2}\right)+T_{2}\left(a_{1}, a_{2}\right)\right]
\end{aligned}
$$

where $T_{1}\left(a_{1}, a_{2}\right)$ and $T_{2}\left(a_{1}, a_{2}\right)$ are defined in Theorem 3.3 , but

$$
\beta_{\alpha}=\int_{0}^{1} \gamma^{\alpha}(1-\gamma)^{\alpha}(d \gamma)^{\alpha}=\frac{\Gamma(1+\alpha)}{\Gamma(1+2 \alpha)}-\frac{\Gamma(1+2 \alpha)}{\Gamma(1+3 \alpha)} .
$$

Proof Since $f_{1}$ and $f_{2}$ are generalized convex on $\left[a_{1}, a_{2}\right]$,

$$
\begin{aligned}
& f_{1}\left(\gamma \frac{a_{1}+a_{2}}{2}+(1-\gamma) x_{2}\right) \leq \gamma^{\alpha} f_{1}\left(\frac{a_{1}+a_{2}}{2}\right)+(1-\gamma)^{\alpha} f_{1}\left(x_{2}\right), \\
& f_{2}\left(\gamma \frac{a_{1}+a_{2}}{2}+(1-\gamma) x_{2}\right) \leq \gamma^{\alpha} f_{2}\left(\frac{a_{1}+a_{2}}{2}\right)+(1-\gamma)^{\alpha} f_{2}\left(x_{2}\right)
\end{aligned}
$$

for all $x_{2} \in\left[a_{1}, a_{2}\right], \gamma \in[0,1]$. Since $f_{1}$ and $f_{1}$ are nonnegative,

$$
\begin{aligned}
f_{1}( & \left.\gamma \frac{a_{1}+a_{2}}{2}+(1-\gamma) x_{2}\right) f_{2}\left(\gamma \frac{a_{1}+a_{2}}{2}+(1-\gamma) x_{2}\right) \\
\quad \leq & \gamma^{2 \alpha} f_{1}\left(\frac{a_{1}+a_{2}}{2}\right) f_{2}\left(\frac{a_{1}+a_{2}}{2}\right)+(1-\gamma)^{2 \alpha} f_{1}\left(x_{2}\right) f_{2}\left(x_{2}\right) \\
& +\gamma^{\alpha}(1-\gamma)^{\alpha} f_{1}\left(\frac{a_{1}+a_{2}}{2}\right) f_{2}\left(x_{2}\right)+\gamma^{\alpha}(1-\gamma)^{\alpha} f_{1}\left(x_{2}\right) f_{2}\left(\frac{a_{1}+a_{2}}{2}\right) .
\end{aligned}
$$

Integrating both sides of the above inequality over $[0,1]$, we obtain

$$
\begin{aligned}
& \frac{1}{\Gamma(1+\alpha)} \int_{0}^{1} f_{1}\left(\gamma \frac{a_{1}+a_{2}}{2}+(1-\gamma) x_{2}\right) f_{2}\left(\gamma \frac{a_{1}+a_{2}}{2}+(1-\gamma) x_{2}\right)(d \gamma)^{\alpha} \\
& \leq \frac{1}{\Gamma(1+\alpha)} f_{1}\left(\frac{a_{1}+a_{2}}{2}\right) f_{2}\left(\frac{a_{1}+a_{2}}{2}\right) \int_{0}^{1} \gamma^{2 \alpha}(d \gamma)^{\alpha} \\
&+\frac{1}{\Gamma(1+\alpha)} f_{1}\left(x_{2}\right) f_{2}\left(x_{2}\right) \int_{0}^{1}(1-\gamma)^{2 \alpha}(d \gamma)^{\alpha} \\
&+\frac{1}{\Gamma(1+\alpha)} f_{1}\left(\frac{a_{1}+a_{2}}{2}\right) f_{2}\left(x_{2}\right) \int_{0}^{1} \gamma^{\alpha}(1-\gamma)^{\alpha}(d \gamma)^{\alpha} \\
&+\frac{1}{\Gamma(1+\alpha)} f_{1}\left(x_{2}\right) f_{2}\left(\frac{a_{1}+a_{2}}{2}\right) \int_{0}^{1} \gamma^{\alpha}(1-\gamma)^{\alpha}(d \gamma)^{\alpha} \\
&= \frac{\Gamma(1+2 \alpha)}{\Gamma(1+3 \alpha)} f_{1}\left(\frac{a_{1}+a_{2}}{2}\right) f_{2}\left(\frac{a_{1}+a_{2}}{2}\right)+\frac{\Gamma(1+2 \alpha)}{\Gamma(1+3 \alpha)} f_{1}\left(x_{2}\right) f_{2}\left(x_{2}\right) \\
&+\frac{1}{\Gamma(1+\alpha)} \beta_{\alpha}\left[f_{1}\left(\frac{a_{1}+a_{2}}{2}\right) f_{2}\left(x_{2}\right)+f_{1}\left(x_{2}\right) f_{2}\left(\frac{a_{1}+a_{2}}{2}\right)\right] .
\end{aligned}
$$


If we integrate both sides of the above inequalities on $\left[a_{1}, a_{2}\right]$, we obtain

$$
\begin{aligned}
& \frac{1}{(\Gamma(1+\alpha))^{2}} \int_{a_{1}}^{a_{2}} \int_{0}^{1} f_{1}\left(\gamma \frac{a_{1}+a_{2}}{2}+(1-\gamma) x_{2}\right) f_{2}\left(\gamma \frac{a_{1}+a_{2}}{2}+(1-\gamma) x_{2}\right)(d \gamma)^{\alpha}\left(d x_{2}\right)^{\alpha} \\
& \leq \frac{\Gamma(1+2 \alpha)}{\Gamma(1+3 \alpha)} \frac{1}{\Gamma(1+\alpha)}\left(a_{2}-a_{1}\right)^{\alpha} f_{1}\left(\frac{a_{1}+a_{2}}{2}\right) f_{2}\left(\frac{a_{1}+a_{2}}{2}\right) \\
&+\frac{\Gamma(1+2 \alpha)}{\Gamma(1+3 \alpha)} \frac{1}{\Gamma(1+\alpha)} \int_{a_{1}}^{a_{2}} f_{1}\left(x_{2}\right) f_{2}\left(x_{2}\right)\left(d x_{2}\right)^{\alpha} \\
&+\frac{1}{(\Gamma(1+\alpha))^{2}} \beta_{\alpha}\left[f_{1}\left(\frac{a_{1}+a_{2}}{2}\right) \int_{a_{1}}^{a_{2}} f_{2}\left(x_{2}\right)\left(d x_{2}\right)^{\alpha}+f_{2}\left(\frac{a_{1}+a_{2}}{2}\right) \int_{a_{1}}^{a_{2}} f_{1}\left(x_{2}\right)\left(d x_{2}\right)^{\alpha}\right] \\
& \leq \frac{\Gamma(1+2 \alpha)}{\Gamma(1+2 \alpha)} \frac{1}{\Gamma(1+\alpha)}\left(a_{2}-a_{1}\right)^{\alpha} \frac{f_{1}\left(a_{1}\right)+f_{1}\left(a_{2}\right)}{2^{\alpha}} \frac{f_{2}\left(a_{1}\right)+f_{2}\left(a_{2}\right)}{2^{\alpha}} \\
&+\frac{\Gamma(1+2 \alpha)}{\Gamma(1+3 \alpha)} \frac{1}{\Gamma(1+\alpha)} \int_{a_{1}}^{a_{2}} f_{1}\left(x_{2}\right) f_{2}\left(x_{2}\right)\left(d x_{2}\right)^{\alpha} \\
&+\frac{1}{(\Gamma(1+\alpha))^{2}} \beta_{\alpha}\left[2^{\alpha} \frac{f_{1}\left(a_{1}\right)+f_{1}\left(a_{2}\right)}{2_{2}} \frac{f_{2}\left(a_{1}\right)+f_{2}\left(a_{2}\right)}{2^{\alpha}}\right]\left(a_{2}-a_{1}\right)^{\alpha} \\
&= \frac{\Gamma(1+2 \alpha)}{\Gamma(1+3 \alpha)} \frac{1}{\Gamma(1+\alpha)} \int_{a_{1}}^{a_{2}} f_{1}\left(x_{2}\right) f_{2}\left(x_{2}\right)\left(d x_{2}\right)^{\alpha}+\frac{1}{2^{\alpha} \Gamma(1+\alpha)}\left[\frac{\Gamma(1+2 \alpha)}{2^{\alpha} \Gamma(1+3 \alpha)}\right. \\
&\left.+\frac{1}{\Gamma(1+\alpha)} \beta_{\alpha}\right]\left(a_{2}-a_{1}\right)^{\alpha}\left[T_{1}\left(a_{1}, a_{2}\right)+T_{2}\left(a_{1}, a_{2}\right)\right] .
\end{aligned}
$$

The proof is complete.

Remark 3.3 If $\alpha=1$, then

$$
\begin{aligned}
& \int_{a_{1}}^{a_{2}} \int_{0}^{1} f_{1}\left(\gamma \frac{a_{1}+a_{2}}{2}+(1-\gamma) x_{2}\right) f_{2}\left(\gamma \frac{a_{1}+a_{2}}{2}+(1-\gamma) x_{2}\right)(d \gamma)\left(d x_{2}\right) \\
& \quad \leq \frac{1}{3} \int_{a_{1}}^{a_{2}} f_{1}\left(x_{2}\right) f_{2}\left(x_{2}\right)\left(d x_{2}\right)+\frac{1}{6}\left(a_{2}-a_{1}\right)\left[T_{1}\left(a_{1}, a_{2}\right)+T_{2}\left(a_{1}, a_{2}\right)\right]
\end{aligned}
$$

\section{Applications}

Example 4.1 Let $a_{1}, a_{2} \in \mathbb{R}_{+}, a_{1}<a_{2}$, and $a_{2}-a_{1} \leq 1$, then

$$
\begin{aligned}
\left\{2^{\alpha}[\right. & \frac{2^{\alpha}}{\Gamma(1+\alpha)}\left(\frac{\Gamma(1+2 \alpha)}{\Gamma(1+3 \alpha)}\right)^{2} \\
& \left.\left.+2^{\alpha}\left(\frac{\Gamma(1+\alpha)}{\Gamma(1+2 \alpha)}-\frac{\Gamma(1+2 \alpha)}{\Gamma(1+3 \alpha)}\right)\left(\frac{\Gamma(1+\alpha)}{\Gamma(1+2 \alpha)}\right)^{2}\right]\right\} K^{2}\left(a_{1}, a_{2}\right) \\
& +\left\{\frac{2^{\alpha}}{\Gamma(1+\alpha)}\left(\frac{\Gamma(1+2 \alpha)}{\Gamma(1+3 \alpha)}\right)^{2}\right. \\
& \left.+4^{\alpha}\left(\frac{\Gamma(1+\alpha)}{\Gamma(1+2 \alpha)}-\frac{\Gamma(1+2 \alpha)}{\Gamma(1+3 \alpha)}\right)\left(\frac{\Gamma(1+\alpha)}{\Gamma(1+2 \alpha)}\right)^{2}\right\} G^{2}\left(a_{1}, a_{2}\right) \\
\leq & 2^{\alpha}\left(\frac{\Gamma(1+2 \alpha)}{\Gamma(1+\alpha) \Gamma(1+3 \alpha)}\right)^{2} \frac{\left(a_{2}^{3 \alpha}-a_{1}^{3 \alpha}\right)}{\left(a_{2}-a_{1}\right)^{\alpha}}+\frac{2^{\alpha}}{(\Gamma(1+\alpha))^{3}} \beta_{\alpha} A^{2}\left(a_{1}, a_{2}\right),
\end{aligned}
$$


where

$$
\begin{aligned}
& \beta_{\alpha}=\int_{0}^{1} \gamma^{\alpha}(1-\gamma)^{\alpha}(d \gamma)^{\alpha}, \\
& A\left(a_{1}, a_{2}\right)=\frac{a_{1}^{\alpha}+a_{2}^{\alpha}}{2^{\alpha}}, \quad a_{1}, a_{2} \geq 0, \\
& G\left(a_{1}, a_{2}\right)=\left(a_{1}^{\alpha} a_{2}^{\alpha}\right)^{\frac{1}{2}}, \quad a_{1}, a_{2} \geq 0,
\end{aligned}
$$

and

$$
K\left(a_{1}, a_{2}\right)=\left(\frac{a_{1}^{2 \alpha}+a_{2}^{2 \alpha}}{2^{\alpha}}\right)^{\frac{1}{2}}, \quad a_{1}, a_{2} \geq 0
$$

Proof If $f_{1} \in G K_{s_{1}}^{2}$ and $f_{2} \in G K_{s_{2}}^{2}$ on $\left[a_{1}, a_{2}\right]$ for some $\gamma \in[0,1]$ and $s_{1}, s_{2} \in(0,1]$, then, by Theorem 3.3, if $f_{1}, f_{2}:[0,1] \longrightarrow\left[0^{\alpha}, 1^{\alpha}\right], f_{1}(x)=x^{\alpha s_{1}}, f_{2}(x)=x^{\alpha s_{2}}$, where $x \in\left[a_{1}, a_{2}\right], s_{1}=s_{2}=$ 1 and $a_{2}-a_{1} \leq 1$, then

$$
\begin{aligned}
& \frac{1}{(\Gamma(1+\alpha))^{3}} \int_{a_{1}}^{a_{2}} \int_{a_{1}}^{a_{2}} \int_{0}^{1}\left(\gamma x_{1}+(1-\gamma) x_{2}\right)^{2 \alpha}(d \gamma)^{\alpha}\left(d x_{2}\right)^{\alpha}\left(d x_{1}\right)^{\alpha} \\
& \leq 2^{\alpha} \frac{\Gamma(1+2 \alpha)}{\Gamma(1+3 \alpha)} \frac{1}{(\Gamma(1+\alpha))^{2}}\left(a_{2}-a_{1}\right)^{\alpha} \int_{a_{1}}^{a_{2}} x_{1}^{2 \alpha}\left(d x_{1}\right)^{\alpha} \\
& +\frac{1}{2^{\alpha}(\Gamma(1+\alpha))^{3}} \beta_{\alpha}\left[a_{1}^{2 \alpha}+a_{2}^{2 \alpha}+2^{\alpha} a_{1}^{\alpha} a_{2}^{\alpha}\right]\left(a_{2}-a_{1}\right)^{2 \alpha} \\
& \Rightarrow \\
& \left(a_{2}-a_{1}\right)^{2 \alpha}\left\{\left[\frac{2^{\alpha}}{\Gamma(1+\alpha)}\left(\frac{\Gamma(1+2 \alpha)}{\Gamma(1+3 \alpha)}\right)^{2}\right.\right. \\
& \left.+2^{\alpha}\left[\frac{\Gamma(1+\alpha)}{\Gamma(1+2 \alpha)}-\frac{\Gamma(1+2 \alpha)}{\Gamma(1+3 \alpha)}\right]\left(\frac{\Gamma(1+\alpha)}{\Gamma(1+2 \alpha)}\right)^{2}\right]\left(a_{1}^{2 \alpha}+a_{2}^{2 \alpha}\right) \\
& \left.+\left[\frac{2^{\alpha}}{\Gamma(1+\alpha)}\left(\frac{\Gamma(1+2 \alpha)}{\Gamma(1+3 \alpha)}\right)^{2}+4^{\alpha}\left[\frac{\Gamma(1+\alpha)}{\Gamma(1+2 \alpha)}-\frac{\Gamma(1+2 \alpha)}{\Gamma(1+3 \alpha)}\right]\left(\frac{\Gamma(1+\alpha)}{\Gamma(1+2 \alpha)}\right)^{2}\right] a_{1}^{\alpha} a_{2}^{\alpha}\right\} \\
& \leq 2^{\alpha}\left(\frac{\Gamma(1+2 \alpha)}{\Gamma(1+\alpha) \Gamma(1+3 \alpha)}\right)^{2}\left(a_{2}-a_{1}\right)^{\alpha}\left(a_{2}^{3 \alpha}-a_{1}^{3 \alpha}\right) \\
& +\frac{1}{2^{\alpha}(\Gamma(1+\alpha))^{3}} \beta_{\alpha}\left(a_{2}-a_{1}\right)^{2 \alpha}\left(a_{2}^{\alpha}+a_{1}^{\alpha}\right)^{2} \\
& \Rightarrow \\
& \left(a_{2}-a_{1}\right)^{2 \alpha}\left\{2 ^ { \alpha } \left[\frac{2^{\alpha}}{\Gamma(1+\alpha)}\left(\frac{\Gamma(1+2 \alpha)}{\Gamma(1+3 \alpha)}\right)^{2}\right.\right. \\
& \left.+2^{\alpha}\left[\frac{\Gamma(1+\alpha)}{\Gamma(1+2 \alpha)}-\frac{\Gamma(1+2 \alpha)}{\Gamma(1+3 \alpha)}\right]\left(\frac{\Gamma(1+\alpha)}{\Gamma(1+2 \alpha)}\right)^{2}\right] K^{2}\left(a_{1}, a_{2}\right) \\
& +\left[\frac{2^{\alpha}}{\Gamma(1+\alpha)}\left(\frac{\Gamma(1+2 \alpha)}{\Gamma(1+3 \alpha)}\right)^{2}\right. \\
& \left.\left.+4^{\alpha}\left[\frac{\Gamma(1+\alpha)}{\Gamma(1+2 \alpha)}-\frac{\Gamma(1+2 \alpha)}{\Gamma(1+3 \alpha)}\right]\left(\frac{\Gamma(1+\alpha)}{\Gamma(1+2 \alpha)}\right)^{2}\right] G^{2}\left(a_{1}, a_{2}\right)\right\}
\end{aligned}
$$




$$
\begin{aligned}
\leq & 2^{\alpha}\left(\frac{\Gamma(1+2 \alpha)}{\Gamma(1+\alpha) \Gamma(1+3 \alpha)}\right)^{2}\left(a_{2}-a_{1}\right)^{\alpha}\left(a_{2}^{3 \alpha}-a_{1}^{3 \alpha}\right) \\
& +\frac{2^{\alpha}}{(\Gamma(1+\alpha))^{3}} \beta_{\alpha}\left(a_{2}-a_{1}\right)^{2 \alpha} A^{2}\left(a_{1}, a_{2}\right) .
\end{aligned}
$$

The proof is complete.

Remark 4.1 If $\alpha=1$, then

$$
\begin{aligned}
& \frac{22}{36} K^{2}\left(a_{1}, a_{2}\right)+\frac{14}{36} G^{2}\left(a_{1}, a_{2}\right) \leq \frac{2}{9}\left(\frac{a_{2}^{3}-a_{1}^{3}}{a_{2}-a_{1}}\right)+\frac{1}{3} A^{2}\left(a_{1}, a_{2}\right) \\
& \quad \Rightarrow \\
& 22 K^{2}\left(a_{1}, a_{2}\right)+14 G^{2}\left(a_{1}, a_{2}\right) \leq 8\left(a_{2}^{2}+a_{1} a_{2}+a_{1}^{2}\right)+12 A^{2}\left(a_{1}, a_{2}\right),
\end{aligned}
$$

where

$$
\begin{aligned}
& A\left(a_{1}, a_{2}\right)=\frac{a_{1}+a_{2}}{2}, \quad a_{1}, a_{2} \geq 0, \text { is the arithmetic mean, } \\
& G\left(a_{1}, a_{2}\right)=\left(a_{1} a_{2}\right)^{\frac{1}{2}}, \quad a_{1}, a_{2} \geq 0, \text { is the geometric mean, and } \\
& K\left(a_{1}, a_{2}\right)=\left(\frac{a_{1}^{2}+a_{2}^{2}}{2}\right)^{\frac{1}{2}}, \quad a_{1}, a_{2} \geq 0, \text { is the quadratic mean. }
\end{aligned}
$$

\section{Competing interests}

The authors declare that they have no competing interests.

\section{Authors' contributions}

Both authors contributed equally in the design and progress of the study. Both authors read and approved the final manuscript.

\section{Author details}

'Department of Mathematics and Institute for Mathematical Research, University Putra Malaysia, Serdang, 43400 , Malaysia. ${ }^{2}$ Department of Mathematics, University Putra Malaysia, Serdang, 43400, Malaysia.

\section{Acknowledgements}

The authors would like to thank the referees for valuable suggestions and comments, which helped the authors to improve this article substantially.

Received: 24 June 2015 Accepted: 15 September 2015 Published online: 06 October 2015

\section{References}

1. Mo, H, Sui, X, Yu, D: Generalized convex functions on fractal sets and two related inequalities. Abstr. Appl. Anal. 2014, Article ID 636751 (2014)

2. Hörmander, L: Notions of Convexity. Springer Science \& Business Media, vol. 127 (2007)

3. Kılıçman, A, Saleh, W: Some inequalities for generalized s-convex functions. JP J. Geom. Topol. 17, 63-82 (2015)

4. Grinblatt, M, Linnainmaa, JT: Jensen's inequality, parameter uncertainty, and multiperiod investment. Rev. Asset Pricing Stud. 1(1), 1-34 (2011)

5. Ruel, JJ, Ayres, MP: Jensen's inequality predicts effects of environmental variation. Trends Ecol. Evol. 14(9), 361-366 (1999)

6. Edgar, GA: Integral, Probability, and Fractal Measures. Springer, New York (1998)

7. Kolwankar, KM, Gangal, AD: Local fractional calculus: a calculus for fractal space-time. In: Fractals: Theory and Applications in Engineering, pp. 171-181. Springer, London (1999)

8. Baleanu, D, Srivastava, HM, Yang, XJ: Local fractional variational iteration algorithms for the parabolic Fokker-Planck equation defined on Cantor sets. Prog. Fract. Differ. Appl. 1(1), 1-11 (2015)

9. Carpinteri, A, Chiaia, B, Cornetti, P: Static-kinematic duality and the principle of virtual work in the mechanics of fractal media. Comput. Methods Appl. Mech. Eng. 191(1-2), 3-19 (2001)

10. Yang, XJ, Baleanu, D, Srivastava, HM: Local fractional similarity solution for the diffusion equation defined on Cantor sets. Appl. Math. Lett. 47, 54-60 (2015)

11. Yang, XJ, Baleanu, D, Srivastava, HM: Local Fractional Integral Transforms and Their Applications. Academic Press, New York (2015) 
12. Zhao, Y, Cheng, DF, Yang, XJ: Approximation solutions for local fractional Schrödinger equation in the on-dimensional Cantorian system. Adv. Math. Phys. 2013, Article ID 5 (2013)

13. Yang, XJ: Advanced Local Fractional Calculus and Its Applications. World Science Publisher, New York (2012)

14. Yang, XJ, Baleanu, D, Machado, JAT: Mathematical aspects of Heisenberg uncertainty principle within local fractional Fourier analysis. Bound. Value Probl. 2013, Article ID 131 (2013)

15. Yang, AM, Chen, ZS, Srivastava, HM, Yang, XJ: Application of the local fractional series expansion method and the variational iteration method to the Helmholtz equation involving local fractional derivative operators. Abstr. Appl. Anal. 2013, Article ID 259125 (2013)

16. Yang, XJ, Baleanu, D, Khan, Y, Mohyud-Din, ST: Local fractional variational iteration method for diffusion and wave equations on Cantor sets. Rom. J. Phys. 59(1-2), 36-48 (2014)

17. Mo, H, Sui, X: Generalized s-convex functions on fractal sets. Abstr. Appl. Anal. 2014, Article ID 254731 (2014)

Submit your manuscript to a SpringerOpen ${ }^{\circ}$ journal and benefit from:

- Convenient online submission

- Rigorous peer review

- Immediate publication on acceptance

- Open access: articles freely available online

- High visibility within the field

- Retaining the copyright to your article 